\title{
SQUID-detected microtesla MRI in the presence of metal
}

\author{
Michael Mößle ${ }^{a}$, Song-I Han ${ }^{b, 1}$, Whittier R. Myers ${ }^{a}$, Seung-Kyun Lee ${ }^{a}$, Nathan Kelso ${ }^{a}$, \\ Michael Hatridge ${ }^{\mathrm{a}}$, Alexander Pines ${ }^{\mathrm{b}}$ and John Clarke $^{\mathrm{a}}$ \\ ${ }^{a}$ Department of Physics, University of California at Berkeley and Lawrence Berkeley \\ National Laboratory \\ ${ }^{\mathrm{b}}$ Department of Chemistry, University of California at Berkeley and Lawrence Berkeley \\ National Laboratory
}

Correspondence Address:

\author{
Michael Moessle \\ Physics Department \\ University of California/Berkeley \\ Berkeley, CA 94720-7300 \\ Phone: (510) 642-3634 Fax: (510) 642-1304 \\ moessle@lbl.gov
}

\footnotetext{
${ }^{1}$ Present address: University of California Santa Barbara, Department of Chemistry and Biochemistry 9510, Santa Barbara, CA 93106
} 


\section{Abstract}

In magnetic resonance imaging (MRI) performed at fields of $1 \mathrm{~T}$ and above, the presence of a metal insert can distort the image because of susceptibility differences within the sample and modification of the radiofrequency fields by screening currents. Furthermore, it is not feasible to perform nuclear magnetic resonance (NMR) spectroscopy or acquire a magnetic resonance image if the sample is enclosed in a metal container. Both problems can be overcome by substantially lowering the NMR frequency. Using a microtesla imaging system operating at $2.8 \mathrm{kHz}$, with a superconducting quantum interference device (SQUID) as the signal detector, we have obtained distortion-free images of a phantom containing a titanium bar and threedimensional images of an object enclosed in an aluminum can; in both cases high-field images are inaccessible.

Keywords: Magnetic resonance imaging, SQUID detection, low field, susceptibility artifacts, RF screening 


\section{Introduction}

In magnetic resonance imaging (MRI) of patients with metallic implants or in imageguided biopsy performed with a metallic needle, one is evidently required to perform MRI in the presence of metal. At the magnetic fields of 1.5 tesla and above used in clinical MRI scanners, metallic objects can not only be a safety hazard [1] for the patient but can produce troublesome artifacts in the MR image $[2,3,4,5,6,7]$. There are two sources of these artifacts: susceptibility differences between the metal and the surrounding tissue, and radiofrequency (RF) screening by the metal. The susceptibility difference between the metal and the surrounding material $[2,4,6]$ causes a local magnetic field inhomogeneity which is proportional to the measurement field $\mathrm{B}_{0}$, resulting in both a shorter relaxation time $\mathrm{T}_{2}{ }^{*}$ and a local change in precession frequency. The reduction in $\mathrm{T}_{2} *$ causes local dephasing, which has a serious effect in gradient echo sequences with long echo times; however, in the spin echo sequences used for all images presented in this paper, this effect is negligible. The local change in precession frequency gives rise to image distortion along the frequency encoding direction. Schenck [4] estimates the maximum spatial distortion due to a susceptibility change $\Delta \chi$ at the boundary between two different materials to be

$$
\Delta \mathrm{x} \approx \Delta \chi \mathrm{B}_{0} / \mathrm{G}_{\mathrm{R}},
$$

where $\mathrm{G}_{\mathrm{R}}$ is the readout gradient. Thus, for a given susceptibility difference $\Delta \chi$, the distortion can be minimized by lowering the measurement field $\mathrm{B}_{0}$ and/or raising the gradient $\mathrm{G}_{\mathrm{R}}$. This reduction in distortion was demonstrated, for example, by Tseng et al. [8] with MRI of hyperpolarized ${ }^{3} \mathrm{He}$ at a measurement field of $2.1 \mathrm{mT}$. 
The second effect of a metal object on nuclear magnetic resonance (NMR) and MRI is its interaction with the RF excitation pulses and the subsequent NMR signal $[5,6,7]$. These pulses induce eddy currents in the metal that generate magnetic screening fields. At frequency $f$ these currents flow in the skin depth

$$
\delta=\left(\pi \mu_{0} \mu \sigma f\right)^{-1 / 2},
$$

the length scale on which an RF field falls to $1 / \mathrm{e}$ of its surface value; $\mu_{0}$ is the permeability of the vacuum and $\mu$ and $\sigma$ are the relative permeability and electrical conductivity of the material. In the case of a piece of metal buried in the sample, the resultant RF inhomogeneity can result in a signal diminuition or enhancement, particularly near the metal [9]. These artifacts dominate over susceptibility artifacts for metals with high conductivity and low susceptibility, for example, copper or aluminum, whereas for materials with low conductivity and high susceptibility such as titanium they are small compared to susceptibility artifacts $[5,6,7]$.

If the sample or part of the sample is completely enclosed in a metal container, screening of both the applied RF field and the detected RF NMR signal is the dominating effect. For example, at the $64 \mathrm{MHz}$ proton frequency of a $1.5 \mathrm{~T}$ MRI system, the skin depth $\delta$ is about $8 \mu \mathrm{m}$ for copper at room temperature, so that even thin pieces of copper screen both the RF pulses and the NMR signal significantly. However, if the field is lowered, for example to $1 \mathrm{mT}$ ( proton frequency $43 \mathrm{kHz}$ ), the skin depth of copper increases to about $0.3 \mathrm{~mm}$, allowing one to image through a correspondingly thicker metallic enclosure. Tseng et al. demonstrated MRI of hyperpolarized ${ }^{3} \mathrm{He}$ through a 25 $\mu \mathrm{m}$ thick brass container at a measurement field of $2.1 \mathrm{mT}$ (frequency $67 \mathrm{kHz}$ for ${ }^{3} \mathrm{He}$ ) [8] and Matlachov et al. [10] obtained a proton NMR spectrum through 2mm-thick 
copper at a measurement field of $2 \mu \mathrm{T}$ (proton frequency $87.9 \mathrm{~Hz}$ ). Minimizing such screening effects is particularly important for applications such as MRI in the presence of metallic vascular stents [9], as well as spectroscopy or imaging in which storage, flow or reactions occur inside metallic pipes, tubes or reaction vessels. Such circumstances are common in situations involving, for example, hazardous materials or extreme physical conditions of temperature and pressure.

Although reducing $\mathrm{B}_{0}$ and thus the NMR frequency reduces artifacts and screening substantially, the signal amplitude in traditional Faraday-detected MRI scales as $\mathrm{B}_{0}{ }^{2}$ in the low-field limit [11]. One approach to improving the SNR at very low fields is to take advantage of the large NMR signal available from hyperpolarized noble gases $[8,12$, 13]. Another method is to prepolarize the sample in a field $B_{p}$ and to perform encoding and detection in a much lower measurement field $\mathrm{B}_{0}[14,15]$; both fields can be generated by copper coils. However, as long as Faraday coil detection is used, the signal amplitude still scales with $\mathrm{B}_{0}$. This loss of sensitivity at low magnetic fields can be circumvented by detecting the signal with a Superconducting QUantum Interference Device (SQUID) [16] coupled to an untuned, superconducting flux transformer. The combination of enhanced polarization and employing SQUID detection results in a SNR that is independent of the measurement field $\mathrm{B}_{0}$, thereby allowing NMR and MRI investigations at very low magnetic fields. For example, prepolarization at 100 to 300 millitesla fields and SQUID detection at $5.6 \mathrm{kHz}$ ( precession field $132 \mu \mathrm{T}$ ) have been used to acquire two-dimensional proton MR images of water and oil phantoms $[17,18]$ and three-dimensional in vivo images of a human forearm and fingers $[19,20]$. 
In this paper we use prepolarization and SQUID-detected MRI to investigate the influence of both metal objects within the sample and nonmagnetic metal enclosures around the sample on the image quality at $66 \mu \mathrm{T}$, and compare the results with images obtained at high magnetic fields ( $>4 \mathrm{~T}$ ).

\section{Experimental configuration}

Our SQUID-detected MRI system $[17,18,19,20]$ is shown schematically in Fig. 1 (a);

Fig.1 (b) shows a typical pulse sequence. First the spins are prepolarized for a time $t_{p}$ in a field $\mathrm{B}_{\mathrm{p}}$ between $40 \mathrm{mT}$ and $100 \mathrm{mT}$ applied along the $x$-direction. As this field is turned off adiabatically, the spins align along the much weaker precession field $\mathrm{B}_{0}$ applied along the $z$-direction. After a delay time $t_{d}$, spin precession is induced using a Hahn-echo sequence. The pulse sequence shown in Fig. 1 (b) is used to obtain three-dimensional images. The precessing proton spins induce an oscillating magnetic field in the lowest coil of a superconducting, second-order axial gradiometer [Fig. 1 (a)]. This gradiometer has a balance of about 1 part in 100 against uniform magnetic fields, thereby reducing external noise from distant sources by a factor of about 100. The gradiometer is connected to a multiturn input coil, integrated onto the SQUID, thus forming a closed superconducting loop. The SQUID is operated in a flux-locked loop [16], the output of which is linear in the applied flux, and yields a magnetic field noise of $1.7 \mathrm{fT} \mathrm{Hz}^{-1 / 2}$ referred to the lowest loop of the gradiometer. An array of Josephson tunnel junctions [18] protects the input circuit from the large transient currents that would otherwise be produced by the polarizing pulse. The SQUID and gradiometer are immersed in liquid helium in a dewar [21] which generates negligible magnetic noise. To attenuate external 
noise further, the entire experiment is enclosed in a 3-mm-thick aluminum shield which attenuates the noise at $2.8 \mathrm{kHz}$ by about a factor of 5 .

The polarizing coil, wound out of braided copper wire to reduce its noise contribution [18], produces a field along the $\mathrm{x}$-direction and is placed below the tail of the dewar with sufficient clearance to accommodate the sample [Fig. 1 (a)]. Three orthogonal pairs of coils, mounted on a 1.8-m wooden cube, produce fields to cancel the Earth's magnetic field. A Helmholtz pair of coils produces the measurement field $\mathrm{B}_{0}$ of $66 \mu \mathrm{T}$ along the $\mathrm{z}$ direction. A pair of coils around the sample space provides the pulsed excitation field $\mathrm{B}_{1}$ along the $\mathrm{x}$-direction. Finally, field gradients along all three spatial directions are generated by Maxwell and Golay coils that are capable of producing gradient field strengths up to about $400 \mu \mathrm{T} / \mathrm{m}$. Detailed specifications of the coils can be found elsewhere $[17,18]$. A Tecmag Orion System generates the necessary pulses for the polarizing, excitation and gradient coils, and also digitizes the signal from the flux-locked loop for subsequent processing.

The comparison images at high field strengths were acquired in the 4.2 tesla field (proton frequency $180 \mathrm{MHz}$ ) of a Nalorac magnet equipped with a Chemagnetics spectrometer and Doty probe and in the 7 tesla field (proton frequency $300 \mathrm{MHz}$ ) of an Oxford magnet equipped with a Varian Unity Inova spectrometer and probe. Both probes are equipped with three orthogonal gradient coils that are capable of producing maximum gradient field strengths of about $700 \mathrm{mT} / \mathrm{m}$ along the $\mathrm{x}-, \mathrm{y}$ - and $\mathrm{z}$-directions. The measurement field is along the axis of the vertical bore magnet, which is defined as the $z$ axis. 


\section{Results and Discussion}

\subsection{Susceptibility artifacts in MRI in the presence of a titanium bar}

To investigate susceptibility artifacts we acquired images of grid-phantoms filled with water containing a $1.5 \mathrm{~mm} \times 10 \mathrm{~mm} \times 15 \mathrm{~mm}$ titanium $\operatorname{bar}\left(\chi_{\mathrm{Ti}} \approx 180 \times 10^{-6}\right)$ [Fig. 2 (a) and (d)] at $7 \mathrm{~T}$ and at $66 \mu \mathrm{T}$. At fields larger than $1.5 \mathrm{~T}$ one expects significantly distorted images $[1,2,6]$ while at $66 \mu \mathrm{T}$ only a very small spatial distortion is expected. To illustrate the susceptibility distortion at high fields, Figs. 2(b) and (c) show images acquired at 7 T. In Fig. 2 (b) the frequency encoding gradient was oriented perpendicular, and in Fig. 2 (c) parallel to the titanium bar. The orientation of the sample relative to the RF coil remained the same. Using the imaging parameters $B_{0}=7 T, G_{R}=$ $40 \mathrm{mT} / \mathrm{m}$ and the susceptibility difference between water and titanium $\Delta \chi=\chi_{\mathrm{Ti}}-\chi_{\text {water }}$ $\approx 190 \times 10^{-6}$, from Eq.(1) we expect a maximum distortion of $3 \mathrm{~mm}$. Given our image resolution of $0.2 \mathrm{~mm}$, we expect severe distortion in both images along the frequency encoding direction, as can be clearly seen in Figs. 2 (b) and (c). The fact that rotating the direction of the encoding gradients by $90^{\circ}$ changed the degree of distortion implies that, as expected for titanium, susceptibility artifacts dominate over RF screening artifacts. Images at $66 \mu \mathrm{T}$ were acquired in the $y z$-plane [Fig. 1 (a)] with the titanium bar aligned along the $\mathrm{y}$-axis and the $\mathrm{B}_{0}$-field aligned along the $\mathrm{z}$-axis perpendicular to the bar. The frequency encoding gradient was along the z-axis and the phase-encoding gradient was along the $\mathrm{y}$-axis. Using the imaging parameters $\mathrm{B}_{0}=66 \mu \mathrm{T}, \mathrm{G}_{\mathrm{R}}=110 \mu \mathrm{T} / \mathrm{m}$ and $\Delta \chi \approx 190 \times 10^{-6}$ we expect a maximum distortion of about $0.3 \mathrm{~mm}$. Figure 2 (e) shows an MR image of the grid phantom [ Fig. 2 (d)] containing the titanium bar. Since the resolution is about $1 \mathrm{~mm}$, as expected the image shows no discernible distortion. 


\subsection{Screening of excitation pulse and MRI signals by metal enclosures}

To investigate the screening of the excitation pulse and the NMR signal we acquired 2D images of phantoms at both microtesla and tesla fields with and without a metal enclosure. The phantom consisted of two columns, one filled with water, the other with $0.5 \%$ agarose gel in water. The pulse sequence for those images is identical to that in Fig. 1 (b), except that for two-dimensional imaging we use only one phase-encoding gradient. Figure 3(a) shows a 2D image of the phantom acquired with $\mathrm{B}_{0}=66 \mu \mathrm{T}$ and $\mathrm{B}_{\mathrm{p}}$ $=150 \mathrm{mT}$. The resolution is $2 \mathrm{~mm}$ and the SNR is 10 . The water appears brighter than the agarose gel due to $T_{1}$ contrast created by using a delay time $t_{d}=300 \mathrm{~ms}$ between turning off the polarizing field $B_{\mathrm{p}}$ and applying the $90^{\circ}$ pulse. During this time interval the magnetization of the agarose gel $\left(T_{1}=250 \mathrm{~ms}\right)$ decays more rapidly than that of the water $\left(\mathrm{T}_{1}=1.5 \mathrm{~s}\right)$ resulting in a weaker NMR signal for the gel [22]. An image of the same sample at $4.2 \mathrm{~T}$ is shown in Fig. 3(b). At this field, the relaxation times are comparable and the two columns show no recognizable difference in intensity. This characteristic difference in $\mathrm{T}_{1}$-weighted contrast at high and low magnetic fields is discussed in detail elsewhere [22].

Low- and high-field images of the same phantom wrapped in $20-\mu \mathrm{m}$-thick aluminum foil are shown in Figs. 3(c) and (d). The image taken at $66 \mu \mathrm{T}$ with the same imaging parameters as in Fig. 3(a) shows the same SNR and resolution as the image with no foil. Since the skin depth of about $2 \mathrm{~mm}$ is much larger than the thickness of the foil, there is no measurable signal attenuation. On the other hand, at $4.2 \mathrm{~T}$ ( proton frequency 180 $\mathrm{MHz}$ ) the skin depth in aluminum is about $8 \mu \mathrm{m}$, resulting in a signal attenuation by a 
factor of about 12. Therefore both the excitation pulse and the signal were substantially attenuated by the foil, and no image is discernible in Fig. 3(d).

To illustrate further the absence of any screening by metal enclosures at very low frequency, we imaged a bare bell pepper and the same pepper enclosed in a metal can. Figure 4(a) shows 6 MRI slices of a bell pepper acquired with the three-dimensional (3D) pulse sequence depicted in Fig. 1(b). The pepper, oriented along the y-direction, was placed in the 50-mm gap between the bottom of the dewar and the polarizing coil. The average polarizing field was $40 \mathrm{mT}$, applied for $500 \mathrm{~ms}$, and the measurement field was $66 \mu \mathrm{T}$ ( proton frequency $2.8 \mathrm{kHz}$ ). The images were acquired in 5 min with a frequency-encoding gradient of $57 \mu \mathrm{T} / \mathrm{m}$ along the $\mathrm{x}$-direction, 31 phase-encoding steps along the z-direction, and 9 phase-encoding steps along the y-direction. The maximum phase-encoding gradient was $100 \mu \mathrm{T} / \mathrm{m}$ along the $\mathrm{z}$-direction and $20 \mu \mathrm{T} / \mathrm{m}$ along the $\mathrm{y}$ direction. The thickness of each section is $8 \mathrm{~mm}$, the SNR is 10 and the in-plane resolution is $2 \mathrm{~mm} \times 2 \mathrm{~mm}$.

Figure 4(b) shows a 3D image of the same pepper, enclosed in a 200- $\mu \mathrm{m}$-thick aluminum can, acquired with the same pulse sequence and experimental parameters. Using Eq. (1), we find that aluminum $\left(\mu=1, \sigma=2 \times 10^{7} \mathrm{~S} / \mathrm{m}\right)$ has a skin depth of $2 \mathrm{~mm}$ at $2.8 \mathrm{kHz}$. Since the skin depth is ten times the thickness of the can, neither the excitation pulse nor the signal from the precessing protons is significantly attenuated by the can. The images of the pepper inside the can show no decrease in signal intensity compared to those of the bare pepper. However, thermal Nyquist currents in the aluminum produce magnetic field noise [23], thereby lowering the SNR of the images by 
a factor of about 1.3. To obtain the image shown in Fig. 4(b) the number of averages was increased from 3 to 4 to achieve the same SNR and resolution as in Fig. 4(a).

\section{Concluding remarks}

At the microtesla imaging fields accessible with SQUID-detected MRI we have shown that the presence of a $1.5 \mathrm{~mm}$ thick titanium bar induces no observable distortion in the image. At high fields, in contrast, the distortion introduced by the titanium bar was severe. At high and intermediate fields these imaging artifacts could be reduced by using a suitable pulse sequence, usually at the expense of measurement time, or adjusting the orientation of the sample relative to the imaging field direction [4]. At microtesla fields, however, the susceptibility artifacts are negligible for any given pulse sequence and sample orientation. Furthermore, we have shown that the image quality at microtesla fields is unaffected when the sample is enclosed in an aluminum can with a wall thickness of $200 \mu \mathrm{m}$, whereas at high fields the sample cannot be accessed at all by NMR imaging.

The fact that the distortion induced by a metal bar is negligible at very low magnetic fields $B_{0}$ for any sample orientation suggests several novel applications. One would be able to obtain distortion-free images in the presence of metallic implants, for example, a knee with an orthopedic titanium screw. Further, assuming one could develop an open microtesla-MRI system, one might hope to be able to image and identify a tumor using the significantly enhanced $T_{1}$-weighted contrast potentially available at low fields [22] and to guide the biopsy of the tumor using a titanium needle. Finally, the imaging of 
samples enclosed in metal containers at microtesla fields may find novel applications across the spectrum from industrial processing to biotechnology.

\section{Acknowledgements}

The authors thank Christian Hilty and Joseph Granwehr for their help with the highfield images. This work was supported by the Director, Office of Science, Office of Basic Energy Sciences, Division of Materials Sciences and Engineering of the U. S. Department of Energy under Contract No. DE-AC03-76SF00098. M. Mößle acknowledges the "Deutsche Forschungsgemeinschaft" for a postdoctoral fellowship.

\section{References}

[1] R. Klucznik, D. Carrier, R. Pyka, R. Haid, Placement of a ferromagnetic intracerebral aneurysm clip in a magnetic field with a fatal outcome, Radiology 187 (1993) $855-6$

[2] J. A. Patton, MR imaging instrumentation and image artifacts, Radiographics 14 (1994) 1083-1096.

[3] K. M. Ludecke, P. Roschmann, R. Tischler, Susceptibility artefacts in NMR imaging, Magn. Reson. Imag. 3 (1985) 329-343.

[4] J. F. Schenck, Role of magnetic susceptibility in MRI, Medical Physics 23 (1996) $815-850$.

[5] C.R. Camacho, D. B. Plewes, R.M. Henkelmann, Nonsusceptibility artifacts due to metallic objects in MR imaging, J. Magn Reson. Imag. 5 (1995) 75-88. 
[6] L. H. Bennett, P. S. Wang, M. J. Donahue, Artifacts in magnetic resonance imaging from metals, J. Appl. Phys. 79 (1996) 4712-4714.

[7] J. A. Malko, J. C. Hoffman Jr, P. J. Jarrett, Eddy-current-induced artifacts caused by an "MR-compatible" halo device, Radiology 173 (1989) 563-564.

[8] C.H. Tseng, G.P. Wong, V.R. Pomeroy, R.W. Mair, D.P. Hinton, D. Hoffmann, R.E. Stoner, F.W. Hersman, D.G. Cory, R.L. Walsworth, Low-field MRI of laser polarized noble gas, Phys. Rev. Lett. 81 (17) (1998) 3785-3788.

[9] H. Graf, G. Steidle, U. Lauer, F. Schick, rf enhancement and shielding in MRI caused by conductive implants: Dependence on electrical parameters for a tube model, Med. Phys. 32 (2005) 337-342.

[10] A. N. Matlachov, P. L. Volegov, M. A. Espy, J. S. George, and R. H. Kraus, SQUID- detected NMR in microtesla magnetic fields, J. Magn. Reson. 170 (2004) $1-4$.

[11] E.M. Haacke, R.W. Brown, M.R. Thompson, R. Venkatesan, Magnetic Resonance Imaging: Physical Principles and Sequence Design, Wiley-Liss, New York, 1999, p. 377

[12] B. Saam, N. Drukker, W. Happer, Edge Enhancement observed with hyperpolarized 3He, Chem. Phys. Lett. 263 (1996) 481-487.

[13] M.P. Augustine, A. Wong-Foy, J.L. Yarger, M. Tomaselli, A. Pines, D.M. Ton That, and J. Clarke, Low field magnetic resonance images of polarized noble gases obtained with a dc superconducting quantum interference device, Appl. Phys. Lett. 72 (1998) 1908-1910. 
[14] J. Stepisink, V. Erzen, and M. Kos, NMR imaging in the earth's magnetic field, Magn. Reson. Med. 15 (1990) 386-391.

[15] A. Macovski and S. Conolly, Novel approaches to low cost MRI, Magn. Reson. Med., 30 (1993) 221-230.

[16] R. Kleiner, D. Koelle, F. Ludwig, and John Clarke, Superconducting quantum interference devices: state of the art and applications, Proceedings of the IEEE 92, (2004) 1534-1548.

[17] R. McDermott, S-K. Lee, B. ten Haken, A. H. Trabesinger, A. Pines, and J. Clarke, Microtesla MRI with a superconducting quantum interference device, Proc. Nat. Acad. Sci. 101 (2004) 7857-7861.

[18] R. McDermott, N. Kelso, S-K. Lee, M. Mößle, M. Mück, W. Myers, B. ten Haken, H.C. Seton, A.H. Trabesinger, A. Pines and J. Clarke, SQUID-detected magnetic resonance imaging in microtesla magnetic fields, J. Low Temp. Phys. 135 (2004) 793-821.

[19] M. Mößle, W.R. Myers, S-K. Lee, N. Kelso, M. Hatridge, A. Pines, John Clarke, SQUID-detected in vivo MRI at microtesla magnetic fields, IEEE Trans. Appl. Supercond. 15 (2005) 757.

[20] M. Mößle, W.R. Myers, S-K. Lee, N. Kelso, M. Hatridge, A. Pines, John Clarke, Microtesla MRI detected with a superconducting quantum interference device, Proc. Intl. Soc. Mag. Res. Med. 13 (2005).

[21] H. C. Seton, J. M. S. Hutchinson, and D. M. Bussel, A 4.2 K receiver coil and SQUID amplifier used to improve the SNR of low-field magnetic resonance images of of the human arm, Meas. Sci. Tech. 8 (1997) 198-207. 
[22] S.-K. Lee, M. Mößle, W.R. Myers, N. Kelso, A. H. Trabesinger, A. Pines, and John Clarke, SQUID-detected MRI at $132 \mu \mathrm{T}$ with T1 contrast weighted at $10 \mu \mathrm{T}-300$ mT, Magn. Reson. Med. 53 (2005) 9-14.

[23] J. R. Clem, Johnson noise from normal metal near a superconducting SQUID gradiometer circuit. IEEE Trans. Magn. 23 (1987) 1093-1096. 


\section{Figure Captions}

Fig. 1. (a) Measurement configuration of SQUID-detected MRI. After the proton spins are prepolarized by a pulsed field $\mathrm{B}_{\mathrm{p}}$, their precession around $\mathrm{B}_{0}$ is detected with a superconducting, second-order gradiometer coupled to a low $\mathrm{T}_{\mathrm{c}}$-SQUID via a 60 -turn $\mathrm{Nb}$ input coil. An array of Josephson junctions limits the maximum current induced in the input circuit during the polarizing pulses. (b) Pulse sequence for three-dimensional imaging. After the polarizing field has been switched off adiabatically, the spins align with the measurement field $\mathrm{B}_{0}$. Following a delay time $\mathrm{t}_{\mathrm{d}}$, spin precession is induced by a standard spin-echo sequence. Spatial encoding in three dimensions is achieved using one frequency- and two phase-encoding gradients.

Fig. 2. Images of water phantoms containing a $1.5 \times 10 \times 15 \mathrm{~mm}$ bar of titanium. (a), (d) Photographs of phantoms. (b), (c) Images acquired at $7 \mathrm{~T}$ and a readout gradient of 40 $\mu \mathrm{T} / \mathrm{m}$ with the bar oriented perpendicular and parallel to the frequency-encoding

direction, respectively. (e) Image acquired at a $132-\mu \mathrm{T}$ measurement field with a readoutgradient of $110 \mu \mathrm{T} / \mathrm{m}$.

Fig. 3. Images of a phantom consisting of water (top) and $0.5 \%$ agarose gel (bottom) at (a) $\mathrm{B}_{0}=66 \mu \mathrm{T}$ and (b) $\mathrm{B}_{0}=4.2 \mathrm{~T}$. Images of the same phantom wrapped in aluminum foil at (c) $\mathrm{B}_{0}=66 \mu \mathrm{T}$ and (d) $\mathrm{B}_{0}=4.2 \mathrm{~T}$. 
Fig. 4. (a) Three-dimensional image of bell pepper showing six cross sections with a thickness of $8 \mathrm{~mm}$; the lines in the photograph mark the position of each slice. (b) Six cross sections of the same pepper enclosed in an aluminum can. In each case, $\mathrm{B}_{0}=66 \mu \mathrm{T}$ and $\mathrm{G}_{\mathrm{freq}}=57 \mu \mathrm{T} / \mathrm{m}$. 


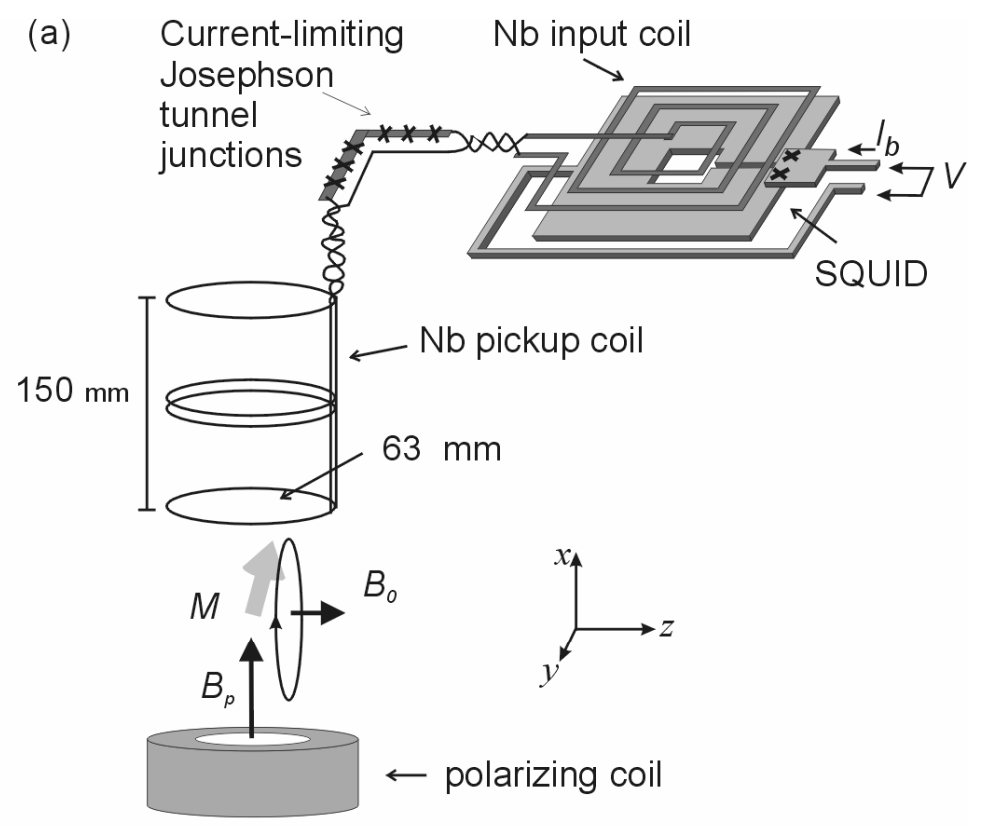

(b)
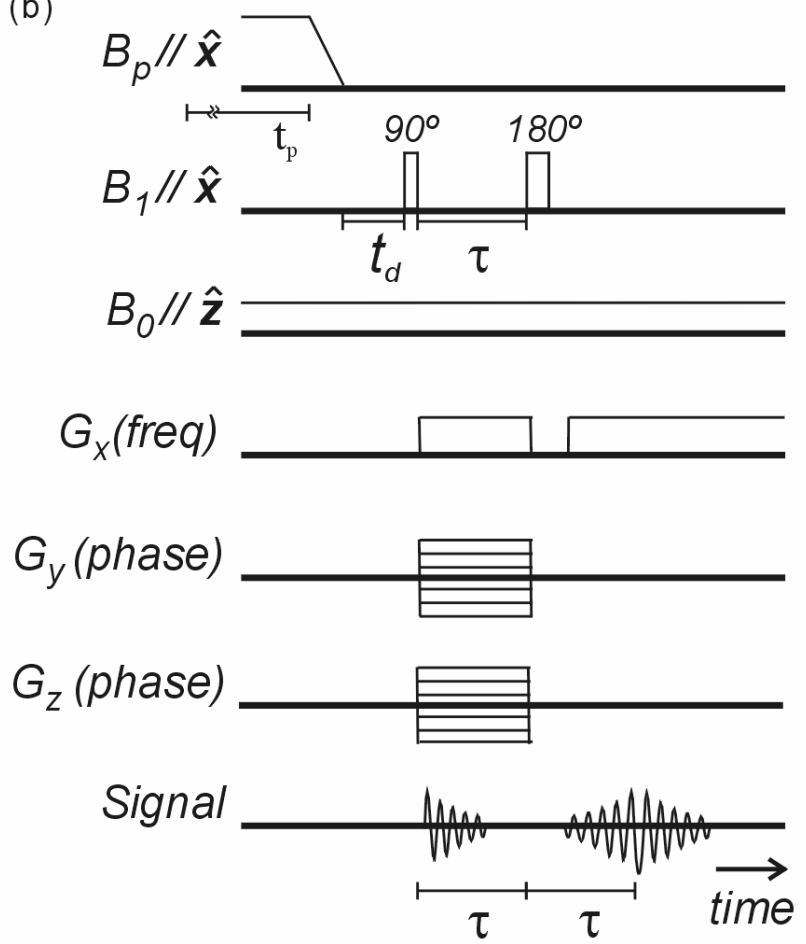

Figure 1 
(a)

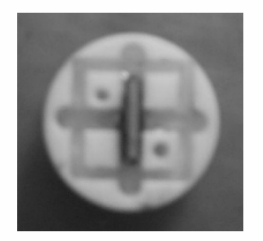

(b)

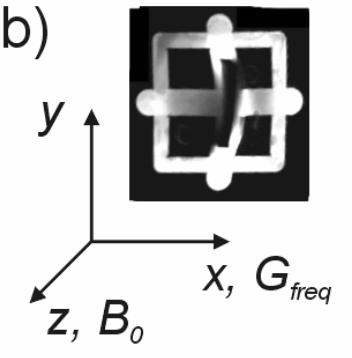

(c)

va

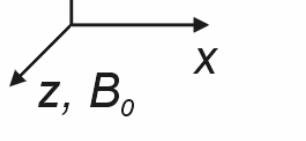

(d)
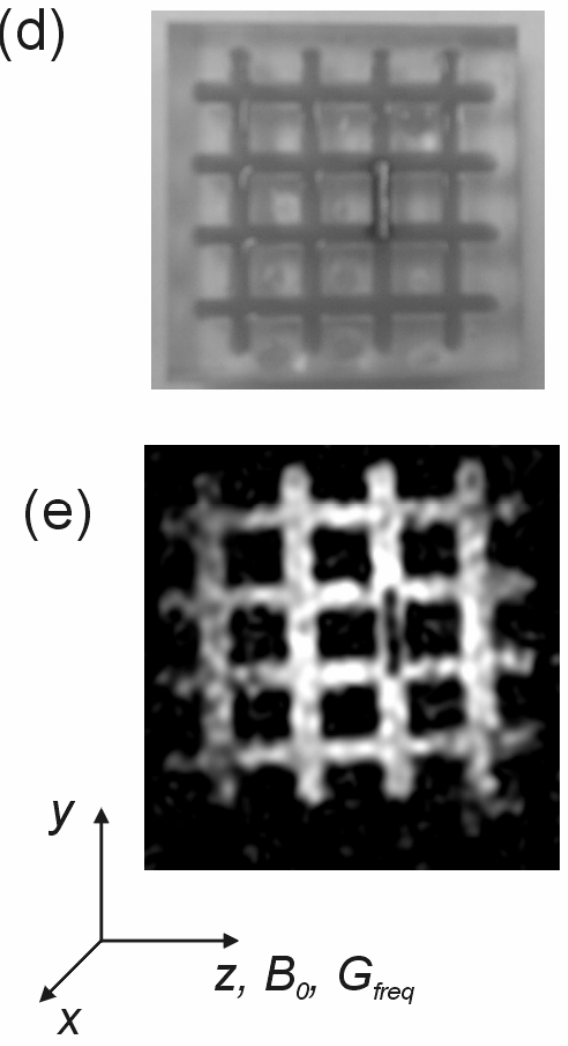

Figure 2 
(a)

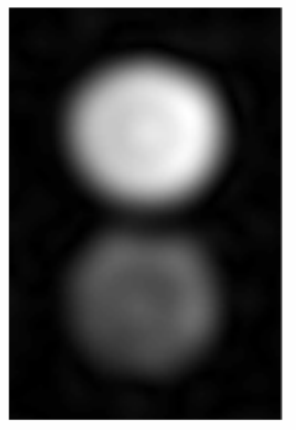

(b)

(c)

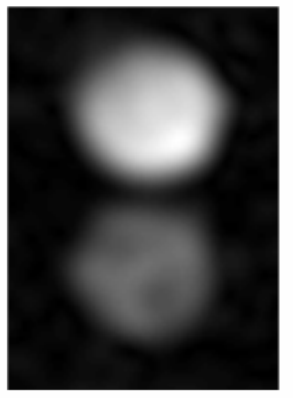

(d)

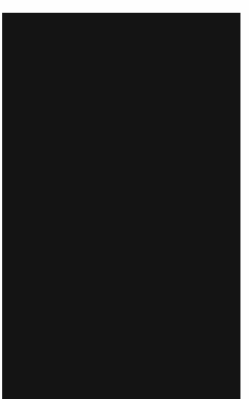

Figure 3 
(a)
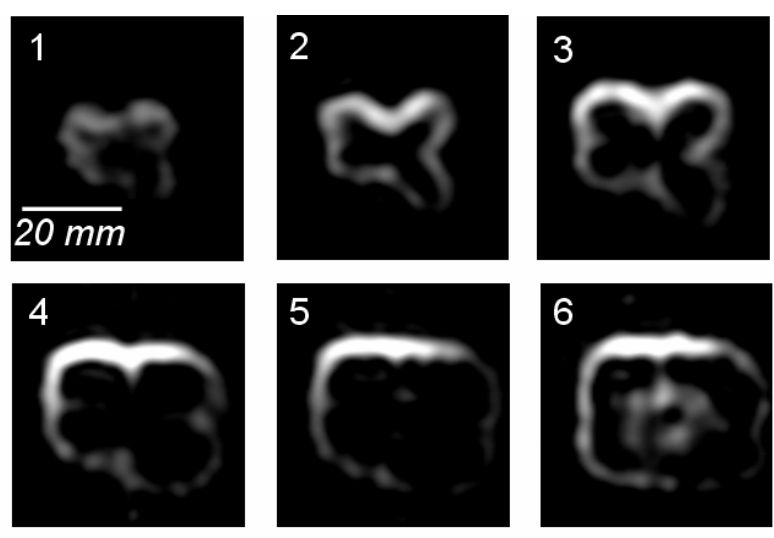

(b)
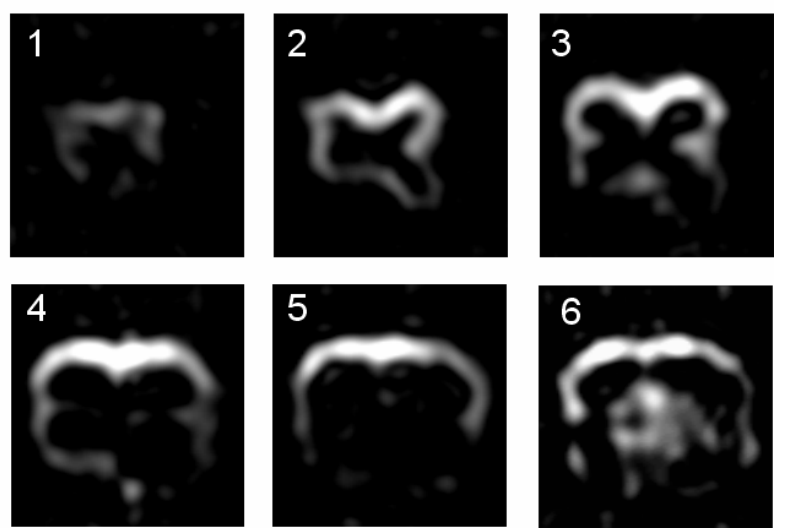

Figure 4 